\title{
Approximate analytical solutions for the relativistic oscillator using a linearized harmonic balance method
}

\author{
A. Beléndez, D. I. Méndez, M. L. Alvarez, C. Pascual and T. Beléndez \\ Departamento de Física, Ingeniería de Sistemas y Teoría de la Señal. \\ Universidad de Alicante. Apartado 99. E-03080 Alicante. SPAIN \\ E-mail: a.belendez@ua.es
}

Corresponding author: A. Beléndez

Phone: +34-96-5903651

Fax: +34-96-5903464

\begin{abstract}
The analytical approximate technique developed by $\mathrm{Wu}$ et al for conservative oscillators with odd non-linearity is used to construct approximate frequency-amplitude relations and periodic solutions to the relativistic oscillator. By combining Newton's method with the method of harmonic balance, analytical approximations to the oscillation period and periodic solutions are constructed for this oscillator. The approximate periods obtained are valid for the complete range of oscillation amplitudes, $A$, and the discrepancy between the second approximate period and the exact one never exceeds $1.24 \%$ and it tends to $1.09 \%$ when $A$ tends to infinity. Excellent agreement of the approximate periods and periodic solutions with the exact ones are demonstrated and discussed.
\end{abstract}

Keywords: Relativistic oscillator; Nonlinear differential equations; Analytical approximation; Harmonic balance method. 
BELÉNDEZ VÁZQUEZ, Augusto, et al. "Approximate analytical solutions for the relativistic oscillator using a linearized harmonic balance method". International Journal of Modern Physics B. Vol. 23, No. 4 (2009). ISSN 0217-9792, pp. 521-536

DOI: $10.1142 / S 0217979209049954$

\section{Introduction}

The study of nonlinear problems is of crucial importance not only in mathematics but also in a lot of areas of physics, engineering and other disciplines, since most phenomena in our world are essentially nonlinear and are described by nonlinear differential equations. It is very difficult to solve nonlinear problems and, in general, it is often more difficult to get an analytic approximation than a numerical one for a given nonlinear problem. In particular, physical and mechanical oscillatory systems are often governed by second order nonlinear differential equations and their study is of great interest to many researchers. There are several methods used to find approximate solutions to nonlinear oscillators, such as perturbation [1,2], variational $[3,4]$, homotopy perturbation [5-11], standard and modified Lindstedt-Poincaré [2,12-18], harmonic balance [2,19-25], bookkeeping parameter [26], iteration perturbation [27], parameter expanding [28], parametrized perturbation [29], artificial parameter [30], linearized and quasilinearized harmonic balance [31-34] methods, etc. Surveys of the literature with numerous references and useful bibliographies may be found in [2,35-37].

As Moreau et al have pointed out [38], a particle undergoing constant acceleration and the simple harmonic oscillator are two elementary topics in classical mechanics that are thoroughly discussed in all of the standard expositions of the subject [39]. But while the relativistic generalization of constant acceleration, defined with respect to instantaneously commoving inertial frames, has received a complete treatment in the literature the relativistic extension of simple harmonic motion, by comparison, is somewhat incomplete. Moreau et al [38] showed that the effect of time dilation along the world line is to cause simple harmonic motion at low energy to become anharmonic at high energy: when the energy of a simple harmonic oscillator is such that the velocities become relativistic, the simple harmonic motion (linear oscillations) at low energy becomes anharmonic (nonlinear oscillations) at high energy [38,40]. Mickens [41] has shown that all the solutions to the relativistic oscillator are periodic and he has determined a method for calculating analytical approximations to its solutions. The purpose of this paper is to determine the high order periodic solutions to the relativistic oscillator by applying the harmonic balance method, which is a procedure for determining analytical 
BELÉNDEZ VÁZQUEZ, Augusto, et al. "Approximate analytical solutions for the relativistic oscillator using a linearized harmonic balance method". International Journal of Modern Physics B. Vol. 23, No. 4 (2009). ISSN 0217-9792, pp. 521-536

DOI: $10.1142 / S 0217979209049954$

approximations to the periodic solutions of differential equations by using a truncated Fourier series. This method, which requires neither a small parameter nor a linear term in the differential equation, yields a very rapid convergence of the solutions series; in most cases only one iteration leads to high accuracy of the solution. This method provides an effective and convenient mathematical tool for nonlinear differential equations.

To applied the harmonic balance method to the relativistic oscillator, we use the analytical approach developed Wu et al [42] which incorporates salient features of both Newton's method and the harmonic balance method. Wu et al's approach is established by successfully linearizing the governing equation and, subsequently, appropriately imposing the harmonic balance method in order to obtain linear algebraic equations, which can be easily solved. Doing this, the complexity of the harmonic balance method is greatly simplified.

\section{The relativistic oscillator}

We consider the relativistic motion of a particle of rest mass $m$ in a onedimensional harmonic oscillator force, $F=-k \bar{x}$, where $k$ is de elastic constant and $\bar{x}$ is the displacement (dimensional variable). Newton's equation of motion can be written in the form

$$
F=\frac{\mathrm{d} p}{\mathrm{~d} \bar{t}}
$$

where $\bar{t}$ is the coordinate time (dimensional variable) and $p$ is the relativistic momentum which can be written as follows

$$
p=\frac{m v}{\sqrt{1-v^{2} / c^{2}}}
$$

where $v=\mathrm{d} \bar{x} / \mathrm{d} \bar{t}$ is the speed of the particle and $c$ is the speed of light. Substituting Eq. (2) 
BELÉNDEZ VÁZQUEZ, Augusto, et al. "Approximate analytical solutions for the relativistic oscillator using a linearized harmonic balance method". International Journal of Modern Physics B. Vol. 23, No. 4 (2009). ISSN 0217-9792, pp. 521-536

DOI: $10.1142 / S 0217979209049954$

into Eq. (1) we obtain

$$
F=\frac{\mathrm{d}}{\mathrm{d} \bar{t}}\left(\frac{m v}{\sqrt{1-v^{2} / c^{2}}}\right)=\frac{m}{\left(1-v^{2} / c^{2}\right)^{3 / 2}} \frac{\mathrm{d} v}{\mathrm{~d} \bar{t}}=\frac{m}{\left[1-\left(1 / c^{2}\right)(\mathrm{d} \bar{x} / \mathrm{d} \bar{t})^{2}\right]^{3 / 2}} \frac{\mathrm{d}^{2} \bar{x}}{\mathrm{~d} \bar{t}^{2}}
$$

Substituting Eq. (3) into Newton's equation of motion in the form

$$
\frac{\mathrm{d} p}{\mathrm{~d} \bar{t}}+k \bar{x}=0
$$

we obtain

$$
\frac{\mathrm{d}^{2} \bar{x}}{\mathrm{~d} \bar{t}^{2}}+\frac{k}{m}\left[1-\frac{1}{c^{2}}\left(\frac{\mathrm{d} \bar{x}}{\mathrm{~d} \bar{t}}\right)^{2}\right]^{3 / 2} \bar{x}=0
$$

From Eq. (5), we can write the governing non-dimensional nonlinear differential equation of motion for the relativistic oscillator as follows

$$
\frac{\mathrm{d}^{2} x}{\mathrm{~d} t^{2}}+\left[1-\left(\frac{\mathrm{d} x}{\mathrm{~d} t}\right)^{2}\right]^{3 / 2} x=0
$$

where $x$ and $t$ are dimensionless variables defined as follows

$$
x=\frac{\omega_{0} \bar{x}}{c} \quad \text { and } \quad t=\omega_{0} \bar{t}
$$

where $\omega_{0}=\sqrt{k / m}$ is the angular frequency for the non-relativistic oscillator (linear oscillator). 
BELÉNDEZ VÁZQUEZ, Augusto, et al. "Approximate analytical solutions for the relativistic oscillator using a linearized harmonic balance method". International Journal of Modern Physics B. Vol. 23, No. 4 (2009). ISSN 0217-9792, pp. 521-536

DOI: $10.1142 / S 0217979209049954$

The even power term in Eq. (6), $(\mathrm{d} x / \mathrm{d} t)^{2}$, acts like the powers of coordinates in that it does not cause a damping of the amplitude of oscillations with time. Therefore, Eq. (6) is an example of a generalized conservative system [2]. At the limit when $(\mathrm{d} x / \mathrm{d} t)^{2}<<1$, Eq. (1) becomes $\left(\mathrm{d}^{2} x / \mathrm{d} t^{2}\right)+x=0$ the oscillator is linear and the proper time $\tau$ becomes equivalent to the coordinate time $t$ to this order.

Introducing the phase space variable $(x, y)$, Eq. (1) can be written in the system form

$$
\frac{\mathrm{d} x}{\mathrm{~d} t}=y, \quad \frac{\mathrm{d} y}{\mathrm{~d} t}=-\left(1-y^{2}\right)^{3 / 2} x
$$

and the trajectories in phase space are given by solutions to the first order, ordinary differential equation

$$
\frac{\mathrm{d} y}{\mathrm{~d} x}=-\frac{\left(1-y^{2}\right)^{3 / 2} x}{y}
$$

As Mickens pointed out, since the physical solutions of both Eq. (6) and Eq. (9) are real, the phase space has a "strip" structure [41], i.e., $-\infty<x<+\infty$ and $-1<y<+1$.

Then unlike the usual non-relativistic harmonic oscillator, the relativistic oscillator is bounded in the y variable. This is due to the fact that the dimensionless variable $y$ is related with the relativistic parameter $\beta=v / c$, where $v$ is the velocity of the particle and $c$ the velocity of light. In the relativistic case, the condition $-c<v<+c$ must be met, and so we obtain $-1<y<+1$. Mickens has proved that all the trajectories to Eq. (9) are closed in the open region of phase space given by $-\infty<x<+\infty$ and $-1<y<+1$, and then all the physical solutions to Eq. (1) are periodic. However, unlike the usual (nonrelativistic) harmonic oscillator, the relativistic oscillator contains higher-order multiples of the fundamental frequency.

The harmonic balance method can now be applied to obtain analytic approximations to the periodic solutions of Eq. (6), we make a change of variable, $y \rightarrow u$, such that $-\infty<u<+\infty$. The required transformation is $[35,40]$ 
BELÉNDEZ VÁZQUEZ, Augusto, et al. "Approximate analytical solutions for the relativistic oscillator using a linearized harmonic balance method". International Journal of Modern Physics B. Vol. 23, No. 4 (2009). ISSN 0217-9792, pp. 521-536

DOI: $10.1142 / S 0217979209049954$

$$
y=\frac{u}{\sqrt{1+u^{2}}}
$$

Introducing a new independent variable $\tau=\omega t$, the corresponding second order differential equation for $u$ is

$$
\omega^{2} u^{\prime \prime}+\frac{u}{\sqrt{1+u^{2}}}=0
$$

where (') denote differentiation with respect to $\tau$. The new independent variable is chosen such that the solution of Eq. (11) is a periodic function of $\tau$ of period $2 \pi$. Eq. (11) is an example of a conservative nonlinear oscillatory system in which the dimensionless restoring force has an irrational form.

We consider the following initial conditions in Eq. (11)

$$
u(0)=B \quad \text { and } \quad u^{\prime}(0)=0
$$

All the motions corresponding to Eq. (5) are periodic [41]; the system will oscillate within symmetric bounds $[-B, B]$, and the angular frequency and corresponding periodic solution of the nonlinear oscillator are dependent on the amplitude $B$.

For small $u$, Eq. (11) approximates that of a linear harmonic oscillator

$$
\omega^{2} u^{\prime \prime}+u \approx 0, \quad \text { for } \quad u<<1
$$

so, for small $B$, the period is $T \approx 2 \pi$. For large $u$ we can consider the following approximation in Eq. (11) 
BELÉNDEZ VÁZQUEZ, Augusto, et al. "Approximate analytical solutions for the relativistic oscillator using a linearized harmonic balance method". International Journal of Modern Physics B. Vol. 23, No. 4 (2009). ISSN 0217-9792, pp. 521-536

DOI: $10.1142 / S 0217979209049954$

$$
\frac{u}{\sqrt{1+u^{2}}} \approx \frac{u}{\sqrt{u^{2}}}=\operatorname{sgn}(u)
$$

where

$$
\operatorname{sgn}(u)=+1, \text { for } u>0 \text { and } \operatorname{sgn}(u)=-1, \text { for } u<0
$$

Then Eq. (11) approximates to the nonlinear oscillator

$$
\omega^{2} u^{\prime \prime}+\operatorname{sgn}(u) \approx 0, \quad \text { for } \quad u>>1
$$

Note that the force corresponding to this system is antisymmetric but piecewise constant. The period for Eq. (16) is $T \approx 4 \sqrt{2 B} \approx 5.6569 \sqrt{B}$ for large $B$ [2]. Consequently the period $T$ increases from $2 \pi$ to $4 \sqrt{2 B}$ as the initial value of $u(0)=B$ increases (between $2 \pi$ to $4 A$ as the oscillation amplitude $A$ for Eq. (11) increases, where $A$ will be related to $B$ in Eq. (46)).

\section{Solution approach}

Eq. (11) is not amenable to exact treatment and, therefore, approximate techniques must be resorted to. There exists no small parameter in Eq. (11), so the usual perturbation methods, i.e. expansion with reference to a centre in a small parameter, cannot be applied. However, we can solve Eq. (11) approximately using the harmonic balance method. To do this, we first write this equation in a form that does not contain the square-root expression

$$
\omega^{2}\left(1+u^{2}\right)\left(u^{\prime \prime}\right)^{2}=u^{2}
$$

Eq. (17) can be rewritten as

$$
\left(1+u^{2}\right)\left(\Omega u^{\prime \prime}\right)^{2}-u^{2}=0
$$


BELÉNDEZ VÁZQUEZ, Augusto, et al. "Approximate analytical solutions for the relativistic oscillator using a linearized harmonic balance method". International Journal of Modern Physics B. Vol. 23, No. 4 (2009). ISSN 0217-9792, pp. 521-536

DOI: $10.1142 / S 0217979209049954$

where $\Omega=\omega^{2}$. The corresponding period of the nonlinear oscillator is given by $T=2 \pi / \sqrt{\Omega}$. Since the restoring force $-u / \sqrt{1+u^{2}}$ is an odd function of $u$, the periodic solution $u(\tau)$ has the following Fourier series representation

$$
u(\tau)=\sum_{n=0}^{\infty} h_{2 n+1} \cos [(2 n+1) \tau]
$$

which contains only odd multiples of $\tau$.

Following the lowest order harmonic balance method, a reasonable and simple initial approximation satisfying the conditions in Eq. (12) would be

$$
u_{1}(\tau)=B \cos \tau
$$

Substituting Eq. (20) into Eq. (18), and expanding the resulting expression in a trigonometric series gives

$$
\left(1+B^{2} \cos ^{2} \tau\right) \Omega^{2} B^{2} \cos ^{2} \tau=B^{2} \cos ^{2} \tau
$$

Expanding and simplifying the above expression gives

$$
\left(\frac{1}{2} \Omega^{2}+\frac{3}{8} \Omega^{2} B^{2}\right) \cos (0 \tau)-\frac{1}{2}+\left(\frac{1}{6} \Omega^{2} B^{2}-\frac{1}{2}\right) \cos 2 \tau+\frac{1}{8} \Omega^{2} B^{2} \cos 4 \tau=0
$$

and setting the coefficient of the resulting term $\cos (0 \tau)$ (the lowest harmonic) equal to zero gives the first analytical approximate value for $\Omega_{1}$ as a function of $B$ 
BELÉNDEZ VÁZQUEZ, Augusto, et al. "Approximate analytical solutions for the relativistic oscillator using a linearized harmonic balance method". International Journal of Modern Physics B. Vol. 23, No. 4 (2009). ISSN 0217-9792, pp. 521-536

DOI: $10.1142 / S 0217979209049954$

$$
\Omega_{1}(B)=\left(1+\frac{3}{4} B^{2}\right)^{-1 / 2}
$$

Therefore, the first analytical approximate period and corresponding periodic solution are

$$
T_{1}(B)=2 \pi / \sqrt{\Omega_{1}(B)} \quad u_{1}(\tau)=B \cos \tau \quad \tau=\sqrt{\Omega_{1}(B)} t
$$

The corresponding approximation to $y$ is gotten from Eq. (10)

$$
y_{1}(t)=\frac{u_{1}(t)}{\sqrt{1+u_{1}^{2}(t)}}=\frac{B \cos \sqrt{\Omega_{1}} t}{\sqrt{1+B^{2} \cos ^{2} \sqrt{\Omega_{1}} t}}
$$

Likewise, $x_{1}(t)$ can be calculated by integrating equation $y=\mathrm{d} x / \mathrm{d} t$ subject to the restrictions

$$
x_{1}(0)=0, \quad y_{1}(0)=\frac{B}{\sqrt{1+B^{2}}}
$$

which can be easily obtain from Eqs. (9) and (10). This integration gives [41]

$$
x_{1}(t)=\left(1+\frac{3}{4} B^{2}\right)^{1 / 4} \sin ^{-1}\left[\frac{B}{\sqrt{1+B^{2}}} \sin \left[\sqrt{\Omega_{1}(B)} t\right]\right]
$$

The harmonic balance method is very difficult to construct higher-order analytical approximations because it requires analytical solutions of sets of complicated nonlinear algebraic equations. To improve this method, $\mathrm{Wu}$ et al [42] presented an approach obtained by combining Newton's method with the harmonic balance method. This method is established by successfully linearizing the governing equation and, subsequently, appropriately imposing the harmonic balance method in order to obtain linear algebraic 
BELÉNDEZ VÁZQUEZ, Augusto, et al. "Approximate analytical solutions for the relativistic oscillator using a linearized harmonic balance method". International Journal of Modern Physics B. Vol. 23, No. 4 (2009). ISSN 0217-9792, pp. 521-536

DOI: $10.1142 / S 0217979209049954$

equations instead of non-linear algebraic equations.

We use $u_{1}(\tau)$ and $\Omega_{1}(B)$ as initial approximations to the solution of Eq. (18), and following Wu et al's method, the first step is the Newton procedure. The periodic solution and the square of frequency of Eq. (18) can be expressed as

$$
u=u_{1}+\Delta u_{1} \quad \Omega=\Omega_{1}+\Delta \Omega_{1}
$$

Substituting Eq. (28) into Eq. (18) and linearizing with respect to the correction terms $\Delta u_{1}$ and $\Delta \Omega_{1}$ lead to

$$
\begin{aligned}
-u_{1}^{2}-2 u_{1} \Delta u_{1}+2 \Omega_{1} u_{1}^{\prime \prime 2} \Delta \Omega_{1}+\Omega_{1}^{2} u_{1}^{\prime \prime 2}+2 \Omega_{1} u_{1}^{2} u_{1}^{\prime \prime 2} \Delta \Omega_{1}+ \\
+\Omega_{1}^{2} u_{1}^{2} u_{1}^{\prime \prime 2}+2 \Omega_{1}^{2} u_{1} u_{1}^{\prime \prime 2} \Delta u_{1}+2 \Omega_{1}^{2} u_{1}^{\prime \prime} \Delta u_{1}^{\prime \prime}+2 \Omega_{1}^{2} u_{1}^{2} u_{1}^{\prime \prime} \Delta u_{1}^{\prime \prime}=0
\end{aligned}
$$

and

$$
\Delta u_{1}(0)=0, \quad \Delta u_{1}^{\prime}(0)=0
$$

where $\Delta u_{1}$ is a periodic function of $\tau$ of period $2 \pi$, and both $\Delta u_{1}$ and $\Delta \Omega_{1}$ are to be determined.

The second approximation to Eq. (29), which must satisfy the initial conditions in Eq. (30), takes de form

$$
\Delta u_{1}(\tau)=c_{1}(\cos \tau-\cos 3 \tau)
$$

where $c_{1}$ is a constant to be determined.

Substituting Eqs. (20) and (31) into Eq. (29), expanding the resulting expression in a trigonometric series and setting the coefficients of the resulting items $\cos (0 \tau)$ and $\cos (2 \tau)$ equal to zero, respectively, yield

$$
\left(B+\frac{3}{4} B^{3}\right) \Omega_{1} \Delta \Omega_{1}+\left(B \Omega_{1}^{2}-B^{2} \Omega_{1}^{2}-1\right) c_{1}-\frac{1}{2} B+\frac{1}{2}\left(1+\frac{3}{4} B^{3}\right) \Omega_{1}^{2}=0
$$

and 
BELÉNDEZ VÁZQUEZ, Augusto, et al. "Approximate analytical solutions for the relativistic oscillator using a linearized harmonic balance method". International Journal of Modern Physics B. Vol. 23, No. 4 (2009). ISSN 0217-9792, pp. 521-536

DOI: $10.1142 / S 0217979209049954$

$$
\left(B-B^{3}\right) \Omega_{1} \Delta \Omega_{1}+\left(8+\frac{11}{2} B^{2}\right) \Omega_{1}^{2} c_{1}+\frac{1}{2} B^{2}-\frac{1}{2}\left(B+B^{3}\right) \Omega_{1}^{2}=0
$$

From Eq. (32) we can obtain $c_{1}$ as follows

$$
c_{1}=\frac{-4 B+\left(8 B+6 B^{3}\right) \Omega_{1} \Delta \Omega_{1}+4 B \Omega_{1}^{2}+3 \Omega_{1}^{2}}{8\left(1-\Omega_{1}^{2}+B^{2} \Omega_{1}^{2}\right)}
$$

Substituting Eq. (34) into Eq. (33), solving for the $\Delta \Omega_{1}$ and taking into account Eq. (23) we obtain

$$
\Delta \Omega_{1}(B)=\frac{7 B^{4}}{\left(128+156 B^{2}+38 B^{4}\right) \sqrt{4+3 B^{2}}}
$$

Furthermore, $c_{1}(B)$ can be obtained by substituting Eq. (35) into Eq. (34) and the result is

$$
c_{1}(B)=\frac{\left(4+3 B^{2}\right) B^{3}}{256+312 B^{2}+76 B^{4}}
$$

The corresponding second analytical approximate periodic solution is given by

$$
T_{2}(B)=2 \pi / \sqrt{\Omega_{2}(B)}, \quad u_{2}(\tau)=\left(B+c_{1}(B)\right) \cos \tau-c_{1}(B) \cos 3 \tau, \quad \tau=\sqrt{\Omega_{2}(B)} t
$$

The corresponding second approximate frequency is

$$
\omega_{2}(B)=\sqrt{\Omega_{2}(B)}=\sqrt{\Omega_{1}(B)+\Delta \Omega_{1}(B)}=\sqrt{\frac{\left(256+312 B^{2}+83 B^{4}\right) \sqrt{4+3 B^{2}}}{512+1008 B^{2}+620 B^{4}+114 B^{6}}}
$$

The corresponding approximation to $y$ is 
BELÉNDEZ VÁZQUEZ, Augusto, et al. "Approximate analytical solutions for the relativistic oscillator using a linearized harmonic balance method". International Journal of Modern Physics B. Vol. 23, No. 4 (2009). ISSN 0217-9792, pp. 521-536

DOI: $10.1142 / S 0217979209049954$

$$
y_{2}(t)=\frac{u_{2}(t)}{\sqrt{1+u_{2}^{2}(t)}}=\frac{B \cos \omega_{2} t+c_{1}\left(\cos \omega_{2} t-\cos 3 \omega_{2} t\right)}{\sqrt{1+\left[B \cos \omega_{2} t+c_{1}\left(\cos \omega_{2} t-\cos 3 \omega_{2} t\right)\right]^{2}}}
$$

However, the analytical integration of Eq. (39) to obtain $x_{2}(t)$ is not possible. To obtain an analytical expression to $x_{2}(t)$, Eq. (39) is written as follows

$$
y_{2}(t)=\frac{B \cos \omega_{2} t+4 c_{1}\left(\cos \omega_{2} t-\cos ^{3} \omega_{2} t\right)}{\sqrt{1+\left[B \cos \omega_{2} t+4 c_{1}\left(\cos \omega_{2} t-\cos ^{3} \omega_{2} t\right)\right]^{2}}}=\frac{B \cos \omega_{2} t+4 c_{1} \cos \omega_{2} t \sin ^{2} \omega_{2} t}{\sqrt{1+\left(B \cos \omega_{2} t+4 c_{1} \cos \omega_{2} t \sin ^{2} \omega_{2} t\right)^{2}}}
$$

The above equation could be easily integrable if $4 c_{1} \cos \omega_{2} t \sin ^{2} \omega_{2} t<<B \cos \omega_{2} t$. Then Eq. (40) would have the same functional form than Eq. (25). From Eq. (36) we can obtain the following limits

$$
\begin{gathered}
\lim _{B \rightarrow 0} \frac{4 c_{1}(B)}{B}=0 \\
\lim _{B \rightarrow \infty} \frac{4 c_{1}(B)}{B}=\frac{4 B^{2}+3 B^{4}}{64+78 B^{2}+19 B^{4}}=\frac{3}{19}=0.1579
\end{gathered}
$$

Then, $4 c_{1}(B) / B$ takes values between 0 to 0.1579 when $B$ varies between 0 and $\infty$. For example, for $B=1,4 c_{1}(B) / B$ takes the value $1 / 23=0.04348$, while for $B=10,4 c_{1}(B) / B$ $=0.1536$. We can write

$$
\left|\frac{4 c_{1}}{B} \cos \omega_{2} t \sin ^{2} \omega_{2} t\right| \leq\left|0.1579 \cos \omega_{2} t \sin ^{2} \omega_{2} t\right| \leq 0.06078
$$

where we have taken into account that the maximum value of $\left|\cos \omega_{2} t \sin ^{2} \omega_{2} t\right|$ is 0.3849 . 
BELÉNDEZ VÁZQUEZ, Augusto, et al. "Approximate analytical solutions for the relativistic oscillator using a linearized harmonic balance method". International Journal of Modern Physics B. Vol. 23, No. 4 (2009). ISSN 0217-9792, pp. 521-536

DOI: $10.1142 / S 0217979209049954$

In Figure 1 we have plotted $\cos z$ and $\cos z \sin ^{2} z$ as a function of $z=\omega_{2} t$. From Eqs. (41)(43) and Figure 1 we can conclude that Eq. (39) can be approximately written as follows

$$
y_{2}(t) \approx \frac{B \cos \omega_{2} t}{\sqrt{1+B^{2} \cos ^{2} \omega_{2} t}}
$$

Later we will verify that such a simple approximation gives very good results for $x(t)$. Likewise, $x_{2}(t)$ can be calculated by integrating equation $y=\mathrm{d} x / \mathrm{d} t$ subject to the restrictions

$$
x_{2}(0)=0, \quad y_{2}(0)=\frac{B}{\sqrt{1+B^{2}}}
$$

and this integration gives

$$
x_{2}(t)=\frac{1}{\omega_{2}(B)} \sin ^{-1}\left[\frac{B}{\sqrt{1+B^{2}}} \sin \left[\omega_{2}(B) t\right]\right]
$$

We will show Eq. (46) gives good results for $x_{2}(t)$.

However, we should not forget that we are really looking for is an approximate analytical solution to Eq. (6), that is, $x(t)$. Moreover, it is convenient to express the approximate angular frequency and the solution in terms of oscillation amplitude $A$ or the amplitude of the dimensionless velocity rather than as a function of $B$. From Eq. (10) we can see that the amplitude of the dimensionless velocity is

$$
y(0)=\frac{v(0)}{c}=\beta_{0}=\frac{B}{\sqrt{1+B^{2}}}
$$

where $v(0)$ and $c$ are the velocities of the particle and the light, respectively. From Eq. (45) 
BELÉNDEZ VÁZQUEZ, Augusto, et al. "Approximate analytical solutions for the relativistic oscillator using a linearized harmonic balance method". International Journal of Modern Physics B. Vol. 23, No. 4 (2009). ISSN 0217-9792, pp. 521-536

DOI: 10.1142/S0217979209049954

we can express $B$ as a function of $\beta_{0}$ as follows

$$
B=\frac{\beta_{0}}{\sqrt{1-\beta_{0}^{2}}}
$$

Now we can find a relation between oscillation amplitude $A$ and parameter $B$ used to solve Eq. (11) approximately. By integrating Eq. (9) and taking into account Eq. (47) we arrive at

$$
\frac{1}{\left(1-y^{2}\right)^{1 / 2}}+\frac{1}{2} x^{2}=\left(1+B^{2}\right)^{1 / 2}
$$

In addition, when $x=A$, the dimensionless velocity $y=\mathrm{d} x / \mathrm{d} t$ is zero and taking this into account in Eq. (49), it follows that

$$
1+\frac{1}{2} A^{2}=\left(1+B^{2}\right)^{1 / 2}
$$

From Eq. (50), we obtain the following relation between amplitude $A$ and parameter $B$

$$
B=A \sqrt{1+\frac{1}{4} A^{2}}
$$

Substituting Eq. (51) in Eqs. (24) and (37) we obtain

$$
T_{1}(A)=2 \pi\left(1+\frac{3}{4} A^{2}+\frac{3}{16} A^{4}\right)^{1 / 4}
$$


BELÉNDEZ VÁZQUEZ, Augusto, et al. "Approximate analytical solutions for the relativistic oscillator using a linearized harmonic balance method". International Journal of Modern Physics B. Vol. 23, No. 4 (2009). ISSN 0217-9792, pp. 521-536

DOI: $10.1142 / S 0217979209049954$

$$
T_{2}(A)=2 \pi\left(\frac{16384+32256 A^{2}+27904 A^{4}+13568 A^{6}+3976 A^{8}+684 A^{10}+57 A^{10}}{\left(4096+4992 A^{2}+2576 A^{4}+664 A^{6}+83 A^{8}\right)\left(16+12 A^{2}+3 A^{4}\right)^{1 / 2}}\right)^{1 / 2}
$$

Taking into account Eq. (48), the first analytical approximate periodic solution for the relativistic oscillator as a function of $\beta_{0}$ is given by

$$
\begin{gathered}
T_{1}\left(\beta_{0}\right)=2 \pi\left(1+\frac{3 \beta_{0}^{2}}{4\left(1-\beta_{0}^{2}\right)}\right)^{1 / 4} \\
x_{1}(t)=\frac{1}{2 \pi} T_{1}\left(\beta_{0}\right) \sin ^{-1}\left[\beta_{0} \sin \left(\frac{2 \pi t}{T_{1}\left(\beta_{0}\right)}\right)\right]
\end{gathered}
$$

Taking into account Eq. (48), the second analytical approximate periodic solution for the relativistic oscillator as a function of $\beta_{0}$ is given by

$$
\begin{gathered}
T_{2}\left(\beta_{0}\right)=2 \pi\left[\frac{\left(10 \beta_{0}^{6}-140 \beta_{0}^{4}+528 \beta_{0}^{2}-512\right)\left(1-\beta_{0}^{2}\right)^{1 / 2}}{\left(27 \beta_{0}^{6}-227 \beta_{0}^{4}+456 \beta_{0}^{2}-256\right)\left(4-\beta_{0}^{2}\right)^{1 / 2}}\right]^{1 / 2} \\
x_{2}(t)=\frac{1}{2 \pi} T_{2}\left(\beta_{0}\right) \sin ^{-1}\left[\beta_{0} \sin \left(\frac{2 \pi t}{T_{2}\left(\beta_{0}\right)}\right)\right]
\end{gathered}
$$

\section{Results and discussion}

In order to illustrate the accuracy and effectiveness of the approach used, we compare the approximate analytical periodic solutions obtained in this paper with the exact ones. For calculating the exact period, $T_{e x}(A)$, we substitute Eq. (51) into Eq. (49) and we obtain 
BELÉNDEZ VÁZQUEZ, Augusto, et al. "Approximate analytical solutions for the relativistic oscillator using a linearized harmonic balance method". International Journal of Modern Physics B. Vol. 23, No. 4 (2009). ISSN 0217-9792, pp. 521-536

DOI: $10.1142 / S 0217979209049954$

$$
\frac{1}{\left(1-y^{2}\right)^{1 / 2}}=\frac{1}{2}\left(A^{2}-x^{2}\right)
$$

From the representation above, we have

$$
T_{e x}(A)=4 \int_{0}^{A} \frac{1+\frac{1}{2}\left(A^{2}-x^{2}\right)}{\sqrt{A^{2}-x^{2}+\frac{1}{4}\left(A^{2}-x^{2}\right)^{2}}} \mathrm{~d} x
$$

which can be written in terms of elliptical integrals as follows

$$
T_{e x}(A)=4 \sqrt{4+A^{2}} E\left(\frac{A^{2}}{4+A^{2}}\right)-\frac{8}{\sqrt{4+A^{2}}} K\left(\frac{A^{2}}{4+A^{2}}\right)
$$

where $K(q)$ and $E(q)$ are the complete elliptic integrals of the first and second kind, respectively, defined as follows [43]

$$
\begin{gathered}
K(q)=\int_{0}^{1} \frac{\mathrm{d} z}{\sqrt{\left(1-z^{2}\right)\left(1-q z^{2}\right)}} \\
E(q)=\int_{0}^{1} \sqrt{\frac{1-q z^{2}}{1-z^{2}}} \mathrm{~d} z
\end{gathered}
$$

For small values of the amplitude $A$ it is possible to take into account the following power series expansion of the exact and approximate periods

$$
T_{e x}(A) \approx 2 \pi\left(1-\frac{3}{16} A^{2}-\frac{15}{1024} A^{4}-\ldots\right)
$$


BELÉNDEZ VÁZQUEZ, Augusto, et al. "Approximate analytical solutions for the relativistic oscillator using a linearized harmonic balance method". International Journal of Modern Physics B. Vol. 23, No. 4 (2009). ISSN 0217-9792, pp. 521-536

DOI: $10.1142 / S 0217979209049954$

$$
\begin{aligned}
& T_{1}(A) \approx 2 \pi\left(1-\frac{3}{16} A^{2}-\frac{6}{1024} A^{4}-\ldots\right) \\
& T_{2}(A) \approx 2 \pi\left(1-\frac{3}{16} A^{2}-\frac{20}{1024} A^{4}-\ldots\right)
\end{aligned}
$$

These series expansions were carried out using MATHEMATICA. As can be seen, in the expansions of the periods $T_{1}$ and $T_{2}$ in powers of $A$, the first two terms are the same as the first two terms of the equation obtained in the power-series expansion of the exact angular frequency, $T_{e x}$. By comparing the third terms in Eqs. (64) and (65) with the third term in the series expansion of the exact period $T_{e x}$ (Eq. (63)), we can see that the third term in the series expansions of $T_{2}$ is more accurate than the third term in the expansion of $T_{1}$. For very large values of the amplitude $A$ it is possible to take into account the following power series expansion of the exact angular frequency

$$
\begin{gathered}
T_{e x}(A) \approx 4 A+\ldots \\
T_{1}(A) \approx 3^{1 / 4} \pi A+\ldots=4.1346 A+\ldots \\
T_{2}(A) \approx 2 \sqrt{\frac{19}{83}} 3^{1 / 4} \pi A+\ldots=3.9564 A+\ldots
\end{gathered}
$$

Once again we can also see than $T_{2}(A)$ provides excellent approximations to the exact period $T_{e x}(A)$ for very large values of oscillation amplitude. Furthermore, we have the following equations

$$
\lim _{A \rightarrow 0} T_{e x}(A)=\lim _{A \rightarrow 0} T_{1}(A)=\lim _{A \rightarrow 0} T_{2}(A)=2 \pi
$$


BELÉNDEZ VÁZQUEZ, Augusto, et al. "Approximate analytical solutions for the relativistic oscillator using a linearized harmonic balance method". International Journal of Modern Physics B. Vol. 23, No. 4 (2009). ISSN 0217-9792, pp. 521-536

DOI: $10.1142 / S 0217979209049954$

$$
\begin{gathered}
\lim _{A \rightarrow \infty} T_{e x}(A)=\lim _{A \rightarrow \infty} T_{1}(A)=\lim _{A \rightarrow \infty} T_{2}(A)=\infty \\
\lim _{A \rightarrow \infty} \frac{T_{1}(A)}{T_{e x}(A)}=1.03364 \\
\lim _{A \rightarrow \infty} \frac{T_{2}(A)}{T_{e x}(A)}=0.98910
\end{gathered}
$$

Eqs. (69)-(72) illustrate very good agreement of the approximate period $T_{2}(A)$ with the exact period $T_{e x}(A)$ for small as well as large values of oscillation amplitude. In Figure 2 we have plotted the percentage error of approximate periods $T_{1}$ and $T_{2}$, as a function of $A$. As we can see from Figure 2, the relative errors for $T_{2}(A)$ are lower than $1.24 \%$ for all the range of values of amplitude of oscillation $A$, and this relative errors tend to $1.09 \%$ when $A$ tends to infinity (see Eq. (72)).

The exact periodic solutions $x(t)$ achieved by numerically integrating Eq. (1), and the proposed approximate periodic solutions $x_{1}(t)$ in Eq. (27) and $x_{2}(t)$ in Eq. (46) are plotted in Figures 3, 4 and 5 for $A=0.1,1$ and $10\left(B=0.10050,1.11803\right.$ and 50.99; $\beta_{0}=$ $0.0996268,0.745356$ and 0.999808$)$, respectively. In these figures parameter $h$ is defined as follows

$$
h=\frac{t}{T_{e x}(A)}
$$

Figures 3-5 show that Eq. (49) provides a good approximation to the exact periodic solutions and that the approximation considered in Eq. (44) is adequate to obtain the approximate analytical expression of $x_{2}(t)$. As we can see, for small values of $A$ (Figure 3) $x(t)$ is very close to the sine function form of non-relativistic simple harmonic motion. For higher values of $A$ the curvature becomes more concentrated at the turning points $(x= \pm A)$. For these values of $A, x(t)$ becomes markedly an-harmonic and is almost straight between the turning points. Only in the vicinity of the turning points, where the magnitude 
BELÉNDEZ VÁZQUEZ, Augusto, et al. "Approximate analytical solutions for the relativistic oscillator using a linearized harmonic balance method". International Journal of Modern Physics B. Vol. 23, No. 4 (2009). ISSN 0217-9792, pp. 521-536

DOI: $10.1142 / S 0217979209049954$

of the Hooke's law force is maximum and the velocity becomes relativistic, is the force effective in changing the velocity [38]. Figure 5 is a typical example of the motion in the ultra-relativistic region where $\beta_{0} \rightarrow 1$.

\section{Conclusions}

A linearized harmonic balance method proposed by $\mathrm{Wu}$ et al [42] was used to obtain two approximate frequencies for the relativistic oscillator. To do this we rewrite the nonlinear differential equation in a form that does not contain an irrational expression. We can conclude that the approximate frequencies obtained are valid for the complete range of oscillation amplitude, including the limiting cases of amplitude approaching zero and infinity. Excellent agreement of the approximate periods with the exact one was demonstrated and discussed and the discrepancy between the second approximate period, $T_{2}$, and the exact one never exceeds $1.24 \%$ and tends to $1.09 \%$ when $A$ tends to infinity. Some examples have been presented to illustrate excellent accuracy of the approximate analytical solutions. Finally, we can see that the method proposed by $\mathrm{Wu}$ et al is very simple in its principle, and is very easy to be applied and it provides very accurate results for the relativistic oscillator.

\section{Acknowledgements}

This work was supported by the "Ministerio de Educación y Ciencia", Spain, under project FIS2005-05881-C02-02. The authors express their gratitude to the reviewer for his useful suggestions and for his comments, which significantly improved the original manuscript. 
BELÉNDEZ VÁZQUEZ, Augusto, et al. "Approximate analytical solutions for the relativistic oscillator using a linearized harmonic balance method". International Journal of Modern Physics B. Vol. 23, No. 4 (2009). ISSN 0217-9792, pp. 521-536

DOI: $10.1142 / S 0217979209049954$

\section{References}

[1] A. H. Nayfeh, Problems in Perturbations (Wiley, New York 1985).

[2] R. E. Mickens, Oscillations in Planar Dynamics Systems (World Scientific, Singapore 1996).

[3] J. H. He, "Determination of limit cycles for strongly nonlinear oscillators" Phys. Rev. Lett. 90, 174301 (2003).

[4] M. D’Acunto, "Determination of limit cycles for a modified van der Pol oscillator", Mech. Res. Commun. 33, 93-98 (2006).

[5] J. H. He, "The homotopy perturbation method for nonlinear oscillators with discontinuities", Appl. Math. Comp. 151, 287-292 (2004).

[6] J. H. He, "Homotopy perturbation method for bifurcation on nonlinear problems", Int. J. Non-linear Sci. Numer. Simulation. 6, 207-208 (2005).

[7] J. H. He, "New interpretation of homotopy perturbation method", Int. J. Mod. Phys. $B, 20,2561-2568$ (2006).

[8] X. C. Cai, W. Y. Wu and M. S. Li, "Approximate period solution for a kind of nonlinear oscillator by He's perturbation method", Int. J. Non-linear Sci. Numer. Simulation 7 (1), 109-117 (2006).

[9] L. Cveticanin, "Homotopy-perturbation for pure nonlinear differential equation", Chaos, Solitons \& Fractals 30, 1221-1230 (2006).

[10] A. Beléndez, A. Hernández, T. Beléndez, C. Neipp and A. Márquez, “Application of the homotopy perturbation method to the nonlinear pendulum", Eur. J. Phys. 28, 93104 (2007).

[11] A. Beléndez, A. Hernández, T. Beléndez, E. Fernández, M. L. Álvarez and C. Neipp, “Application of He's homotopy perturbation method to the Duffing-Harmonic 
BELÉNDEZ VÁZQUEZ, Augusto, et al. "Approximate analytical solutions for the relativistic oscillator using a linearized harmonic balance method". International Journal of Modern Physics B. Vol. 23, No. 4 (2009). ISSN 0217-9792, pp. 521-536

DOI: $10.1142 / S 0217979209049954$

oscillator", Int. J. Non-linear Sci. Numer. Simulation 8, 79-88 (2007).

[12] J. H. He, "Modified Lindstedt-Poincare methods for some non-linear oscillations. Part I: expansion of a constant”, Int. J. Non-linear Mech. 37, 309-314 (2002).

[13] J. H. He, "Modified Lindstedt-Poincare methods for some non-linear oscillations. Part II: a new transformation", Int. J. Non-linear Mech. 37, 315-320 (2002).

[14] J. H. He, "Modified Lindstedt-Poincare methods for some non-linear oscillations. Part III: double series expansion”, Int. J. Non-linear Sci. Numer. Simulation 2, 317$320(2001)$.

[15] P. Amore and A. Aranda, "Presenting a new method for the solution of nonlinear problems", Phys. Lett. A 316, 218-225 (2003).

[16] G. M. Abd El-Latif, "On a problem of modified Lindstedt-Poinaré for certain strongly non-linear oscillators", Appl. Math. Comput. 152 821-836 (2004).

[17] P. Amore, A. Raya and F. M. Fernández, "Alternative perturbation approaches in classical mechanics", Eur. J. Phys. 26, 1057-1063 (2005).

[18] J. I. Ramos, “On Lindstedt-Poincaré techniques for the quintic Duffing equation", Appl. Math. Comput. (2007), doi:10.1016/ j.amc.2007.03.050.

[19] R. E. Mickens, "Comments on the method of harmonic-balance", J. Sound. Vib. 94, 456-460 (1984).

[20] R. E. Mickens, "Mathematical and numerical study of the Duffing-harmonic oscillator", J. Sound Vib. 244, 563-567 (2001).

[21] G. R. Itovich and J. L. Moiola, "On period doubling bifurcations of cycles and the harmonic balance method", Chaos, Solitons \& Fractals 27, 647-665 (2005).

[22] H. Hu and J. H. Tang, "Solution of a Duffing-harmonic oscillator by the method of harmonic balance", J. Sound Vib. 294, 637-639 (2006). 
BELÉNDEZ VÁZQUEZ, Augusto, et al. "Approximate analytical solutions for the relativistic oscillator using a linearized harmonic balance method". International Journal of Modern Physics B. Vol. 23, No. 4 (2009). ISSN 0217-9792, pp. 521-536

DOI: $10.1142 / S 0217979209049954$

[23] A. Beléndez, A. Hernández, A. Márquez, T. Beléndez and C. Neipp, “Analytical approximations for the period of a simple pendulum", Eur. J. Phys. 27, 539-551 (2006).

[24] H. Hu, "Solution of a quadratic nonlinear oscillator by the method of harmonic balance", J. Sound Vib. 293, 462-468 (2006).

[25] A. Beléndez, A. Hernández, T. Beléndez, M. L. Álvarez, S. Gallego, M. Ortuño and C. Neipp, "Application of the harmonic balance method to a nonlinear oscillator typified by a mass attached to a stretched wire", J. Sound Vib. 302, 1018-1029 (2007).

[26] J. H. He, "Bookkeeping parameter in perturbation methods", Int. J. Non-linear Sci. Numer. Simulation 2, 257-264 (2001).

[27] J. H. He, "Iteration perturbation method for strongly nonlinear oscillators", $J$. Vibration Control 7, 631-642 (2001).

[28] D. H. Shou and J. H. He, "Application of parameter-expanding method to strongly nonlinear oscillators", Int. J. Non-linear Sci. Numer. Simulation 8, 121-124 (2007).

[29] J. H. He, "Some new approaches to Duffing equation with strongly and high order nonlinearity (II) parametrized perturbation technique", Commun. Nonlinear Sci. Numer. Simulation 4(1), 81-83 (1999).

[30] J. H. He, "A modified perturbation technique depending upon an artificial parameter”, Meccanica 35 (4), 299-311 (2000).

[31] C. W. Lim and B. S. Wu, "A new analytical approach to the Duffing-harmonic oscilla-tor", Phys. Lett.A 311, 365-373 (2003).

[32] B. S. Wu and C. W. Lim, "Large amplitude nonlinear oscillations of a general conservative system", Int. J. Non-linear Mech. 39, 859-870 (2004).

[33] C. W. Lim and B. S. Wu, "Accurate higher-order approximations to frequencies of nonlinear oscillators with fractional powers", J. Sound Vib. 281, 1157-1162 (2005). 
BELÉNDEZ VÁZQUEZ, Augusto, et al. "Approximate analytical solutions for the relativistic oscillator using a linearized harmonic balance method". International Journal of Modern Physics B. Vol. 23, No. 4 (2009). ISSN 0217-9792, pp. 521-536

DOI: $10.1142 / S 0217979209049954$

[34] C. W. Lim, B. S. Wu and W. P. Sun, "Higher accuracy analytical approximations to the Duffing-harmonic oscillator", J. Sound Vib. 296, 1039-1045 (2006).

[35] J. H. He, Non-perturbative methods for strongly nonlinear problems (dissertation.deVerlag im Internet GmbH, Berlin 2006).

[36] J. H. He, "Some asymptotic methods for strongly nonlinear equations", Int. J. Mod. Phys. B, 20, 1141-1199 (2006).

[37] L. Xu, "He's parameter-expanding methods for strongly nonlinear oscillators", $J$. Comput. Appl. Math. (2006), doi:10.1016/j.cam.2006. 07.020.

[38] W. Moreau, R. Easther and R. Neutze, "Relativistic (an)harmonic oscillator", Am. J. Phys. 62, 531-535 (1994).

[39] J. L. Synge, Classical Dynamics in Encyclopeadia of Physics, edited by S. Flügge (Springer, Berlin, 1960), Vol. III/1, pp. 210-211.

[40] H. Goldstein, Classical Mechanics, $2^{\text {nd }}$ ed. (Addison-Wesley, Reading, MA, 1981).

[41] R. E. Mickens, "Periodic solutions of the relativistic harmonic oscillator", J. Sound Vib. 212, 905-908 (1998).

[42] B. S. Wu, W. P. Sun and C. W. Lim, "An analytical approximate technique for a class of strongly non-linear oscillators", Int. J. Non-linear Mech. 41, 766-774 (2006).

[43] L. M. Milne-Thomson, "Elliptic integrals" in M. Abramowitz and I. A. Stegun (Eds.), Handbook of Mathematical Functions (Dover Publications, Inc., New York, 1972). 
BELÉNDEZ VÁZQUEZ, Augusto, et al. "Approximate analytical solutions for the relativistic oscillator using a linearized harmonic balance method". International Journal of Modern Physics B. Vol. 23, No. 4 (2009). ISSN 0217-9792, pp. 521-536

DOI: $10.1142 / S 0217979209049954$

\section{FIGURE CAPTIONS}

Figure 1. $\cos z$ and $\cos z \sin ^{2} z$ as a function of $z=\omega_{2} t$.

Figure 2. Relative error for approximate periods $T_{1}$ (dashed line) and $T_{2}$ (continuous line).

Figure 3. Comparison of the normalized approximate analytical solutions $x_{1} / A(\bullet)$ and $x_{2} / A$ (O) with the exact solution (continuous line) for $A=0.1$ $\left(\beta_{0}=v_{0} / c=0.09963\right)$.

Figure 4. Comparison of the normalized approximate analytical solutions $x_{1} / A(\bullet)$ and $x_{2} / A$ (O) with the exact solution (continuous line) for $A=1$ $\left(\beta_{0}=v_{0} / c=0.74536\right)$.

Figure 5. Comparison of the normalized approximate analytical solutions $x_{1} / A(\bullet)$ and $x_{2} / A$ (○) with the exact solution (continuous line) $A=10$ $\left(\beta_{0}=v_{0} / c=0.99981\right)$. 
Figure 1

FIGURE 1

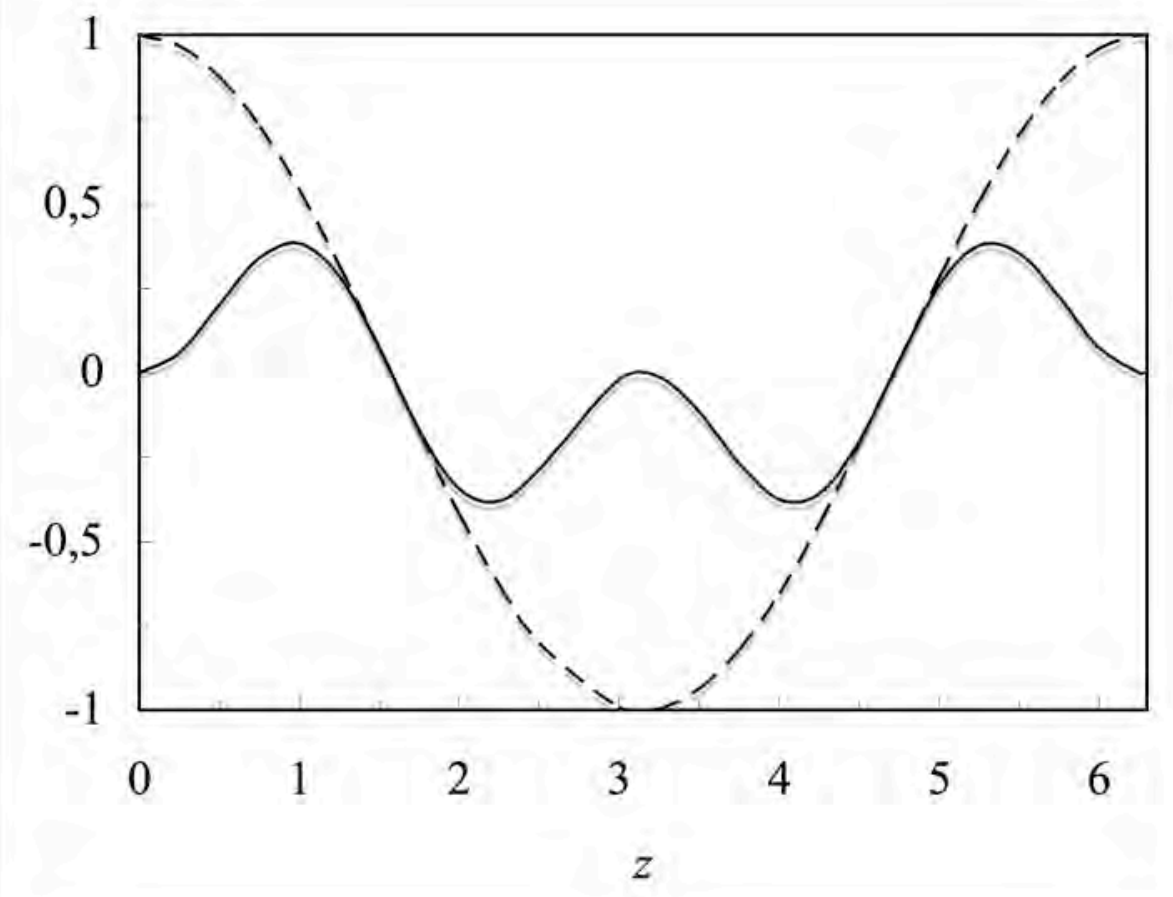


FIGURE 2

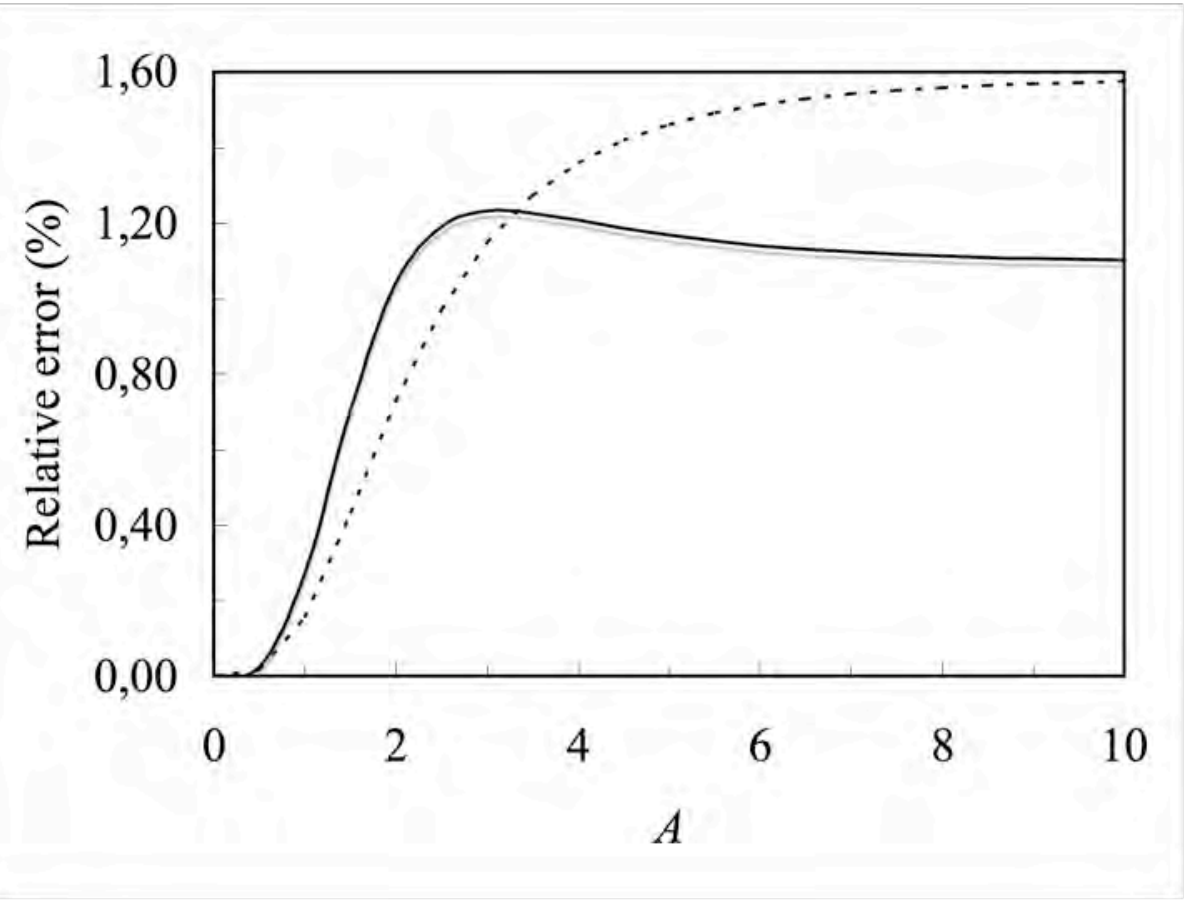

Figure 2 
FIGURE 3

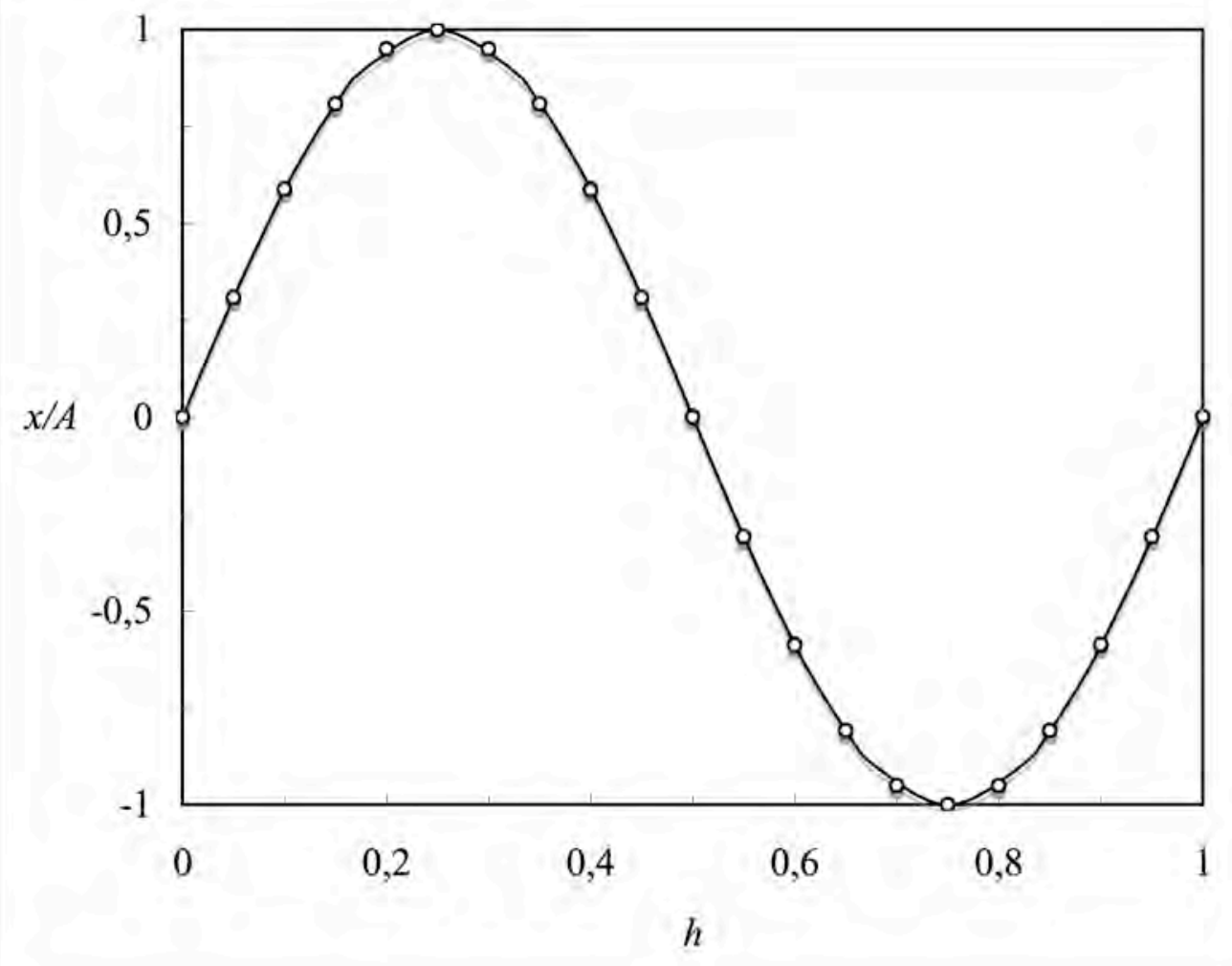


Figure 4

FIGURE 4

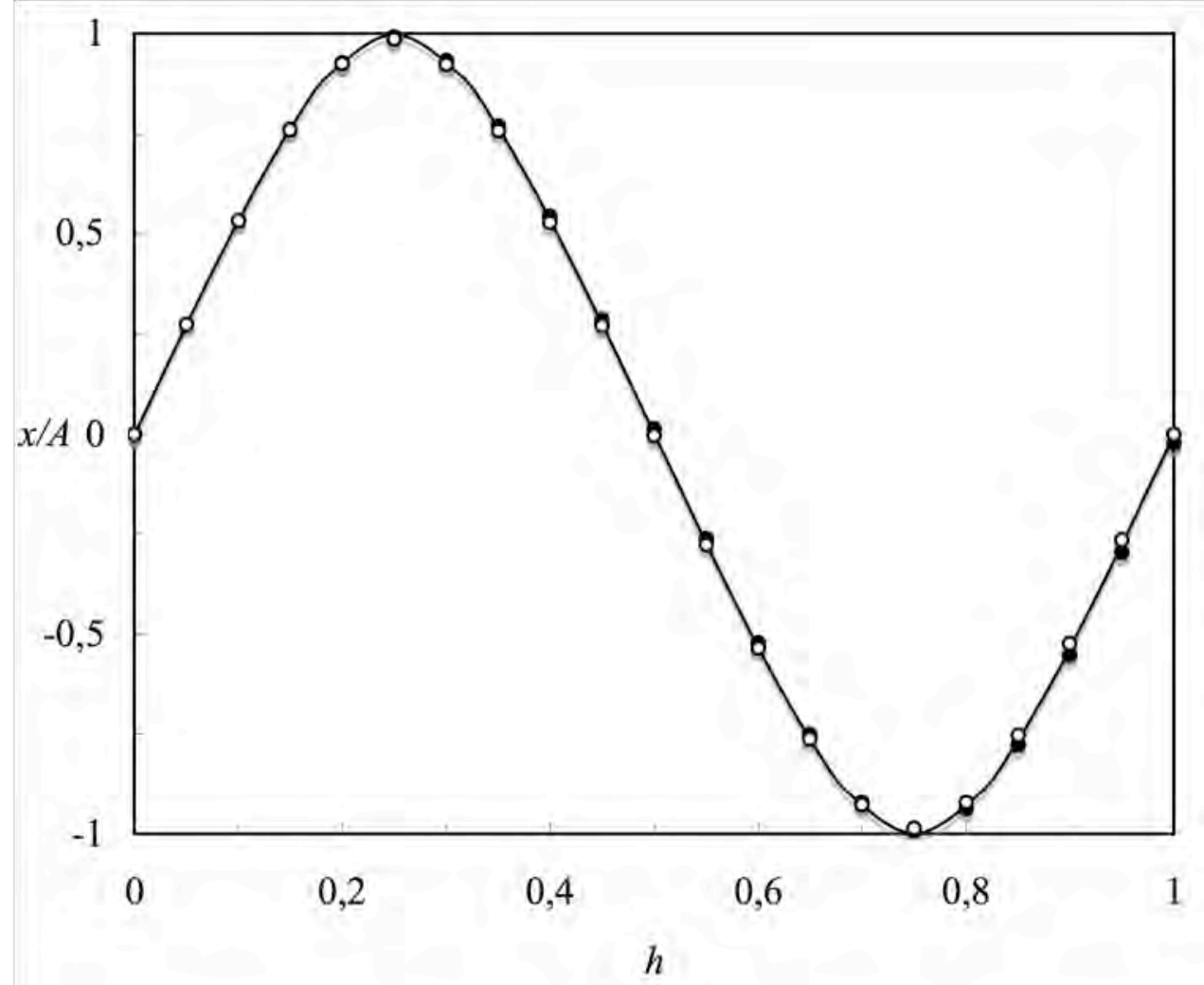


FIGURE 5

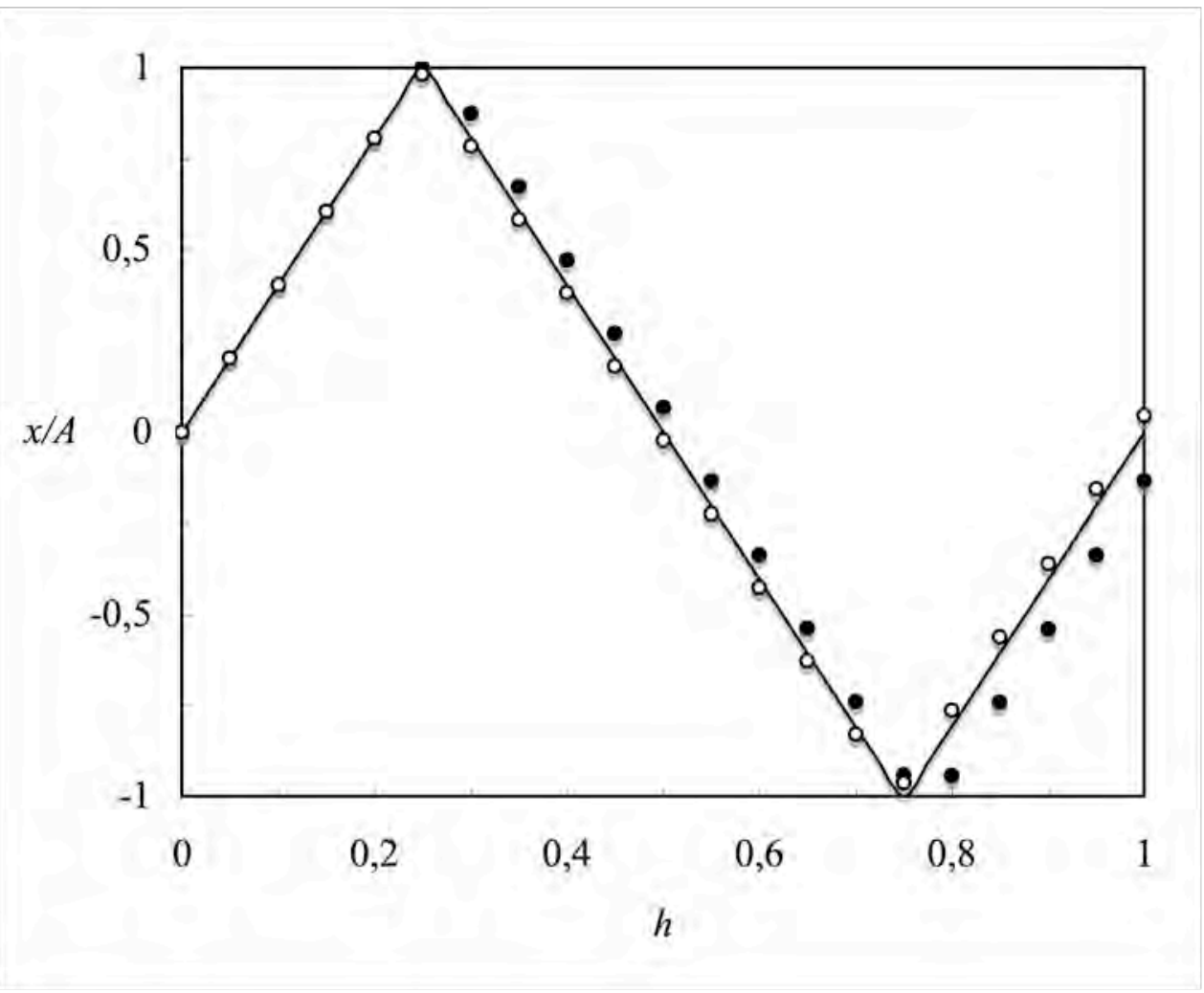

Figure 5 